\section{Validade da informação sobre o peso ao nascer para estudos fundamentados na programação fetal}

\section{Validity of information on birth weight for studies based on fetal programming}

\section{Validez de la información sobre el peso al nacer para estudios basados en la programación fetal}

\section{Resumo}

Peso ao nascer é uma informação essencial nos estudos de programação fetal e, em geral, obtida retrospectivamente. No Sistema de Informações sobre Nascidos Vivos (SINASC), peso ao nascer é uma informação válida, mas nem sempre acessível. Objetivou-se estabelecer um algoritmo para seleção da fonte de dados de maior confiabilidade do peso ao nascer na ausência da informação do SINASC. No estudo seccional de estudantes de 6-14 anos, Niterói, Rio de Janeiro, Brasil, 2010, peso ao nascer foi coletado por meio de questionário de autopreenchimento, entrevista, prontuários do Programa Médico de Família (PMF), além do SINASC. Foram calculados coeficientes de correlação intraclasse (CCI) e diferenças das médias de peso ao nascer. CCIs variaram de 0,90 a 0,99. Todas as fontes apresentaram médias de peso ao nascer maiores do que SINASC, considerando-se aceitáveis diferenças até 100g. O peso ao nascer é registrado no nascimento (SINASC) ou próximo deste (PMF) e, na ausência dessas fontes, a lembrança do peso ao nascer de 6-14 anos após o nascimento é uma opção confiável. Para complementar a informação sobre peso ao nascer na ausência do SINASC, recomenda-se a seguinte ordenação: $P M F$, entrevista e questionário.

Peso ao Nascer; Reprodutibilidade dos Testes; Sistemas de Informação; Desenvolvimento Fetal
Gisele Almeida de Noronha 1

Pauline Lorena Kale 1,2

Tania Zdenka Guillén de Torres 1,2

Antonio Jose Leal Costa 1,2

Maria de Lourdes Tavares Cavalcanti 1,2

Moyses Szklo 1,2

doi: 10.1590/0102-311X00051816
Correspondência

G. A. Noronha

Instituto de Estudos em Saúde Coletiva, Universidade Federal do Rio de Janeiro.

Av. Horácio Macedo s/n, Rio de Janeiro, RJ 21941-598, Brasil. giselenoronha@gmail.com

1 Instituto de Estudos em Saúde Coletiva, Universidade Federal do Rio de Janeiro, Rio de Janeiro, Brasil.

2 Faculdade de Medicina, Universidade Federal do Rio de Janeiro, Rio de Janeiro, Brasil. 


\section{Introdução}

Desde o final da década de 1980, a partir da hipótese da Programação Intrauterina para Doenças da Vida Adulta ou hipótese de Barker 1, a relação entre as condições de vida intrauterina e a saúde no ciclo da vida vem sendo estudada. Há fortes evidências de que o crescimento intrauterino restrito (CIUR) faz com que o feto desenvolva um metabolismo econômico (hipótese do "Fenótipo Econômico") e que essa programação vantajosa para sua sobrevivência intraútero pode ser deletéria mediante uma dieta rica em calorias ao longo da vida ${ }^{2}$. Acredita-se que esses danos in utero podem causar alterações no metabolismo do indivíduo, provocando doenças crônicas em longo prazo, como excesso de peso, diabetes, hipertensão e doenças coronarianas 3 .

Embora não represente exatamente o fenômeno do CIUR, cuja identificação necessitaria de seguimento durante a gestação 4, o pequeno para idade gestacional (PIG), de mais fácil aferição, tem sido empregado como um marcador de CIUR. Um marcador simplificado de CIUR, mais utilizado quando os dados sobre peso ao nascer e idade gestacional são categóricos, considera aqueles nascidos com peso inferior a $2.500 \mathrm{~g}$ (baixo peso ao nascer) e com 37 semanas ou mais de gestação (não prematuros) 5 . A identificação do CIUR ainda é controversa e complexa na prática, já que exige dados fidedignos do peso ao nascer e da idade gestacional 6 . Na ausência de informação válida sobre idade gestacional, somente o peso ao nascer exato ou categórico são utilizados nos estudos de programação fetal.

Estudos epidemiológicos no ciclo da vida preferencialmente usam informações prospectivas do peso ao nascer, entretanto dados retrospectivos são mais facilmente obtidos, permitindo análises mais rápidas 7 . Sendo assim, diferentes fontes de dados secundários vêm sendo utilizadas, seja com base em registros de nascimento e de acompanhamento de crianças na infância ou prontuários médicos, seja de forma recordatória, dependente, portanto, da memória do indivíduo entrevistado 8,9 .

A validade das informações recordatórias referentes ao início da vida do indivíduo varia com o tempo e deve considerar a possibilidade de viés de informação 10,11. Erros de classificação do peso ao nascer recordatório, 11 anos após o nascimento, estiveram relacionados à baixa escolaridade materna e à ausência de parceiro ${ }^{7}$.

Quando a informação sobre o peso ao nascer, essencial nos estudos epidemiológicos de programação fetal, é obtida retrospectivamente a partir de diferentes fontes de dados e de forma complementar, tornam-se imprescindíveis estudos de confiabilidade e validade.

Em pesquisa fundamentada na hipótese da programação fetal realizada com estudantes de 6-14 anos de escolas públicas de Niterói, Rio de Janeiro, Brasil 12, o peso ao nascer foi obtido de diferentes formas para garantir o máximo de completude da informação: por meio de um questionário de autopreenchimento, entrevista, consulta a prontuários médicos de puericultura do Programa Médico de Família (PMF) e registros oficiais de nascimento do Sistema de Informações sobre Nascidos Vivos (SINASC/Ministério da Saúde). A informação sobre o peso ao nascer do SINASC é considerada válida e confiável 13,14,15,16.

O objetivo do presente estudo é avaliar a validade da informação sobre o peso ao nascer de estudantes de 6-14 anos de escolas públicas de Niterói, 2010, obtida a partir de diversas fontes de dados, com a finalidade de estabelecer um algoritmo para seleção da fonte de informação de maior validade, na ausência da informação do SINASC.

\section{Métodos}

O presente estudo está vinculado a pesquisa interinstitucional (Universidade Federal do Rio de Janeiro - UFRJ, Universidade Federal Fluminense - UFF e Universidade Estadual do Rio de Janeiro - UERJ). Trata-se de um estudo seccional de estudantes de 6-14 anos de duas escolas públicas localizadas no Município de Niterói, em 2010. A pesquisa foi conduzida em três etapas: 1a etapa: inquérito nutricional (795 estudantes) realizado em duas escolas municipais que oferecem turmas do primeiro ao nono ano do ensino fundamental, localizadas em áreas de menor risco de violência no município e que são adscritas ao PMF há pelo menos 14 anos; 2a etapa: constituída por um estudo caso-controle (362 estudantes; razão de casos e controles de 1:2) no qual os casos foram definidos como tendo excesso de peso (escore Z de índice de massa corporal - IMC para idade e sexo $>+1,00$ ), 
identificados no inquérito nutricional, e os controles foram selecionados de forma aleatória a partir de todos os escolares participantes da primeira etapa, estratificados por faixa etária (6-9 e 10-14 anos) e escola; $3^{\text {a }}$ etapa, incluindo todos os participantes do inquérito nutricional, caracterizada por coleta de dados secundários em prontuários médicos de puericultura do PMF (224 prontuários recuperados) e no SINASC da Secretaria de Saúde do Estado do Rio de Janeiro. As bases de dados da pesquisa e do SINASC foram relacionadas probabilisticamente (386 pares de registros). Informações mais detalhadas da pesquisa podem ser obtidas em outra publicação 12.

Em 2010, Niterói apresentava uma população estimada de 487.562 habitantes, a menor taxa de analfabetismo (2,3\%) (Departamento de Informática do SUS. Informações de saúde: demográficas e socioeconômicas, 2010. http://www2.datasus.gov.br/DATASUS/index.php?area=02), o maior Índice de Desenvolvimento Humano $(\mathrm{IDH}=0,886)$ e a maior renda per capita domiciliar $(\mathrm{R} \$ 1.938,00)$ do Estado do Rio de Janeiro, embora a distribuição de renda fosse desigual (coeficiente de Gini = 0,598) (Serviço Brasileiro de Apoio às Micro e Pequenas Empresas. Informações socioeconômicas do Município de Niterói. http://www.bis.sebrae.com.br/bis/conteudoPublicacao.zhtml?id+3906, acessado em 13/Set/2014).

No início da década de 1990, foram implantados o SINASC do Ministério da Saúde 17,18 e o PMF 19, em Niterói. O SINASC tem cobertura universal, e as informações perinatais são coletadas no momento do nascimento 18. O PMF atende aos princípios e às diretrizes do Sistema Único de Saúde (SUS) e foi implantado prioritariamente em áreas de maior risco social e ambiental de Niterói, em 1992, privilegiando munícipes com renda familiar mensal inferior a cinco salários mínimos. O PMF de Niterói serviu de modelo para outros projetos de saúde da família no próprio estado e, posteriormente, para outros estados brasileiros, até que em 1997, o Ministério da Saúde recomendou o PMF, como uma estratégia de reorientação do modelo assistencial para todo o território nacional 19.

\section{Estudo de validade da informação sobre peso ao nascer}

\section{- Fontes de informações sobre peso ao nascer exato}

A informação do peso ao nascer exato foi coletada de diferentes formas. Para os 795 estudantes participantes do inquérito escolar, as fontes de informação do peso ao nascer exato foram SINASC, PMF e questionário de autopreenchimento. A completude da informação foi, respectivamente, de 49\%, $18,5 \%$ e $74 \%$. Adicionalmente, para os 362 estudantes do inquérito escolar selecionados para o estudo caso-controle, foi realizada entrevista com $89 \%$ de obtenção da informação do peso ao nascer. Informações recordatórias do peso ao nascer do questionário de autopreenchimento (86\%) e da entrevista (85\%) foram preferencialmente fornecidas pela mãe biológica. Cerca de 13\% dos estudantes (105) não tinham nenhuma informação sobre o peso ao nascer exato. Esse grupo tem um percentual maior de escolares com 10-14 anos em relação ao grupo com, pelo menos, uma fonte de informação, mas não difere em relação ao sexo e à escola de origem.

As fontes de informações foram consideradas pelos pesquisadores, a priori, de melhor para pior validade da informação sobre o peso ao nascer: (1) SINASC (registro oficial da informação, no momento do nascimento); (2) prontuário médico do PMF (informações coletadas por profissionais da saúde na puericultura, próximas ao nascimento); (3) entrevista preferencialmente à mãe do escolar na segunda etapa da pesquisa; e (4) informação obtida por meio de questionário de autopreenchimento pelos responsáveis do estudante no inquérito escolar, na primeira etapa da pesquisa.

\section{Amostra analítica}

Todos os estudantes que participaram da primeira etapa da pesquisa (795) eram elegíveis para o estudo de validade; 690 estudantes apresentaram, pelo menos, um registro do peso ao nascer exato; 40 estudantes apresentaram o registro do peso ao nascer exato nas quatro fontes de informação avaliadas.

Para algumas análises, foi avaliado o subgrupo dos participantes que tinham informações completas sobre o peso ao nascer, isto é, escolares que apresentaram completude nas quatro fontes de informação sobre o peso ao nascer $(n=40)$. 


\section{Análise estatística}

Inicialmente, os grupos sem informação sobre o peso ao nascer exato foram comparados com aqueles que apresentaram informações de, pelo menos, uma fonte segundo sexo, faixa etária e escola, utilizando-se o teste do qui-quadrado.

Em seguida, foi realizada uma análise exploratória do peso ao nascer exato por fonte de informação, a partir de estatísticas sumárias e gráficos box-plot. Além disso, foram calculadas as diferenças absolutas do peso ao nascer de cada fonte de informação em relação ao SINASC. Foi considerado aceitável um valor absoluto menor que $100 \mathrm{~g}$ de diferença entre as fontes de informação 20.

Para a análise de validade do peso ao nascer exato do SINASC e cada uma das demais fontes de informações, foi utilizada inicialmente a abordagem gráfica de confiabilidade de Bland e Altman para investigar as relações entre os erros de medição e o verdadeiro valor das médias 21 obtido pelos dados do SINASC. Valores atípicos (observações fora dos limites de concordância: média das diferenças \pm 2 desvios-padrão) foram identificados e excluídos. A seguir, foram feitos gráficos de dispersão do peso ao nascer e, a fim de calcular concordâncias absolutas, calculados coeficientes de correlação intraclasse (CCI) e respectivos intervalos de 95\% de confiança (IC95\%), usando o modelo de dois fatores com fonte de informação fixa (two-way mixed-effect-model) 22,23. Considerou-se que os estudantes foram selecionados aleatoriamente a partir de uma população de referência e as respectivas fontes de informação sobre o peso ao nascer são fixas. Foi utilizada a seguinte categorização para o coeficiente de correlação intraclasse: 0,21-0,40 (fraca); 0,41-0,60 (moderada); 0,61-0,80 (substancial); 0,81-0,99 (quase perfeita) e 1,00 (perfeita) 24 .

Para avaliar a presença de erros sistemáticos, foi ajustado o modelo de dois fatores com fonte de informação fixa (two-way mixed-effect-model), considerando o peso ao nascer como desfecho e fonte de informação como variável explicativa. Foram testadas as diferenças entre as médias de peso ao nascer exato por pares de cada uma das fontes de informação em relação ao SINASC. A qualidade do modelo foi avaliada pela análise dos resíduos, excluindo-se valores atípicos (desvios padronizados $\geq+3$ e $\leq-3$ ).

Posteriormente, na ausência da informação do SINASC, os dados do peso ao nascer foram sendo completados a partir das demais fontes de informação que apresentaram maior confiabilidade, obedecendo, portanto, à ordenação decrescente do CCI.

As análises foram realizadas utilizando-se o programa Stata, versão 12.0 (StataCorp LP, College Station, Estados Unidos).

\section{Considerações éticas}

A pesquisa foi aprovada pelo Comitê de Ética em Pesquisa do Instituto de Estudos em Saúde Coletiva, Universidade Federal do Rio de Janeiro (parecer no 77-2008). Os dados foram coletados mediante assinatura de Termo de Consentimento Livre e Esclarecido pelos responsáveis e pelos estudantes de 10-14 anos de idade.

\section{Resultados}

No inquérito nutricional, dos 795 estudantes avaliados, aproximadamente $87 \%$ tinham o peso ao nascer exato de, pelo menos, uma fonte de informação. Não houve diferença estatisticamente significante para as variáveis sexo e escola. Adolescentes de 10-14 anos apresentaram maior proporção de ausência de informação quando comparados às crianças de 6-9 anos de idade $(16,1 \%$ e 8,1\%; valor de p = 0,001).

As estatísticas sumárias do peso ao nascer exato, segundo fonte de informação, são apresentadas na Figura 1. Observa-se que as fontes de dados do SINASC e do PMF apresentaram menor variabilidade e menor frequência de valores atípicos. Os valores mínimo e máximo de peso ao nascer foram observados nas fontes de informação, respectivamente, questionário de autopreenchimento (700g) e entrevista $(4.950 \mathrm{~g})$. A menor média de peso ao nascer correspondeu à fonte de informação PMF, enquanto a maior média foi por entrevista.

Analisando as diferenças de peso ao nascer exato entre o SINASC e cada uma das demais fontes de informação (Tabela 1), em 73,2\% dos casos, o peso ao nascer coletado no PMF e no SINASC foram 


\section{Figura 1}

Estatísticas sumárias do peso ao nascer, segundo fonte de informação, dos escolares de 6-14 anos. Niterói, Rio de Janeiro, Brasil, 2010.

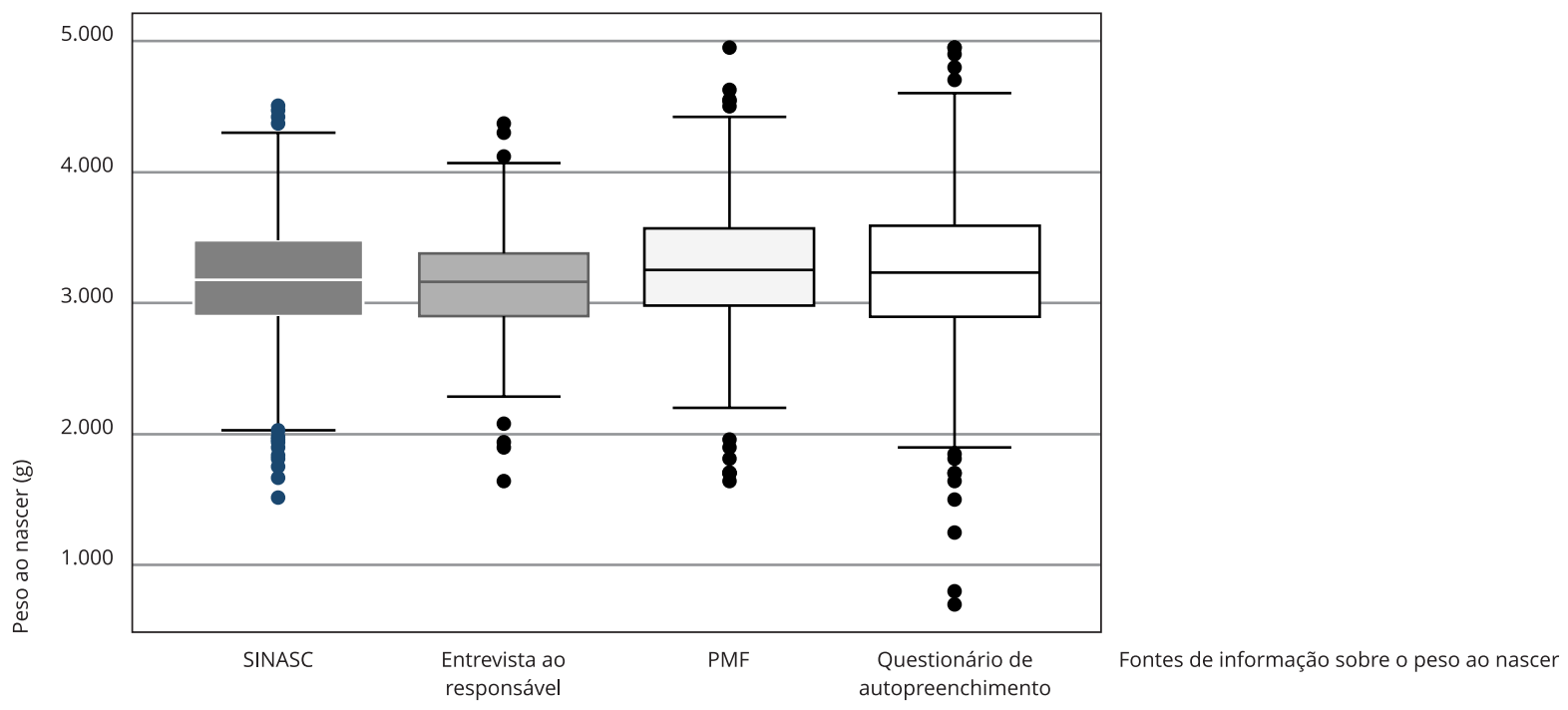

PMF: Programa Médico de Família; SINASC: Sistema de Informações sobre Nascidos Vivos.

Tabela 1

Distribuição das diferenças de peso ao nascer, entre as fontes de informações, dos escolares de 6-14 anos. Niterói, Rio de Janeiro, Brasil, 2010.

\begin{tabular}{|c|c|c|c|c|c|c|c|c|c|}
\hline \multirow{3}{*}{$\begin{array}{l}\text { Diferenças do peso } \\
\text { ao nascer }(\mathrm{g})\end{array}$} & \multicolumn{9}{|c|}{ Fontes de informação } \\
\hline & \multicolumn{3}{|c|}{ PMF - SINASC } & \multicolumn{3}{|c|}{ Entrevista - SINASC } & \multicolumn{3}{|c|}{ Questionário - SINASC } \\
\hline & $\mathbf{n}$ & $\%$ & $\%$ & $\mathbf{n}$ & $\%$ & $\%$ & $\mathbf{n}$ & $\%$ & $\%$ \\
\hline-500 ou mais & 1 & 1,4 & & 6 & 3,2 & & 20 & 6,0 & \\
\hline-499 a 250 & 3 & 4,2 & 7,0 & 1 & 0,5 & 7,4 & 11 & 3,3 & 15,6 \\
\hline$-249 a-100$ & 1 & 1,4 & & 7 & 3,7 & & 21 & 6,3 & \\
\hline$-99 a-1$ & 2 & 2,8 & & 6 & 3,2 & & 26 & 7,8 & \\
\hline Nenhuma diferença & 52 & 73,2 & 83,1 & 126 & 66,7 & 76,2 & 138 & 41,4 & 58,9 \\
\hline 1 a 99 & 5 & 7,0 & & 12 & 6,3 & & 32 & 9,6 & \\
\hline 100 a 249 & 3 & 4,2 & & 17 & 9,0 & & 28 & 8,4 & \\
\hline 250 a 499 & 1 & 1,4 & 9,9 & 6 & 3,2 & 16,4 & 26 & 7,8 & 25,5 \\
\hline 500 ou mais & 3 & 4,2 & & 8 & 4,2 & & 31 & 9,3 & \\
\hline Total & 71 & 100,0 & & 189 & 100,0 & & 333 & 100,0 & \\
\hline
\end{tabular}

PMF: Programa Médico de Família; SINASC: Sistema de Informações sobre Nascidos Vivos.

zero e, em $83,1 \%$, a diferença foi de \pm 0 a $99 \mathrm{~g}$. Na comparação do peso ao nascer entre as fontes, questionário de autopreenchimento e SINASC, apenas 58,9\% dos pares tiveram diferenças absolutas inferiores a $100 \mathrm{~g}$, enquanto essas diferenças absolutas de $\pm 99 \mathrm{~g}$ ocorreram em cerca de $76 \%$, quando os dados da entrevista foram comparados aos do SINASC. Nas diferenças iguais ou superiores a 100g, predominam valores positivos, sugerindo superestimação do peso ao nascer das três fontes em relação ao SINASC. 
A Figura 2 apresenta os gráficos com abordagem Bland-Altman para todos os escolares e aqueles com informações completas do peso ao nascer exato nas quatro fontes de informações. Em ambos os grupos, a maioria das observações faz referência às diferenças nulas, equivalente aos pontos na linha zero. Dentro da faixa de concordância, observam-se valores dispersos, sem um padrão, o que sugere ausência de viés sistemático. Fora da faixa de concordância, ocorreram alguns valores atípicos, mais frequentes no grupo total (de 5-21 casos) quando comparado ao grupo com as quatro fontes de informações (de 1-4 casos). Os gráficos referentes ao SINASC e ao questionário de autopreenchimento (terceiro gráfico de cada linha) apresentaram maior amplitude da faixa que inclui 95\% das diferenças (faixa de concordância) e maior frequência de valores atípicos nos dois grupos (todos os escolares: 21 casos; e escolares com informações completas: 4 casos) (Figura 2).

Nos gráficos de dispersão do peso ao nascer do SINASC e em cada uma das três demais fontes de informação, o grupo com todos os escolares apresentou mais valores dispersos em torno da linha diagonal (representando concordância perfeita) quando comparados ao grupo dos estudantes com informações completas (Figura 3). A concordância do peso ao nascer do SINASC com cada uma das três fontes de informação foi igual ou superior a 0,90, isto é, concordância quase perfeita nos dois grupos de estudantes avaliados (Figura 3).

\section{Figura 2}

Abordagem gráfica de Bland-Altman comparando as fontes de informação de peso ao nascer dos escolares de 6-14 anos. Niterói, Rio de Janeiro, Brasil, 2010

2a) Todos os escolares com, pelo menos, uma fonte de informação

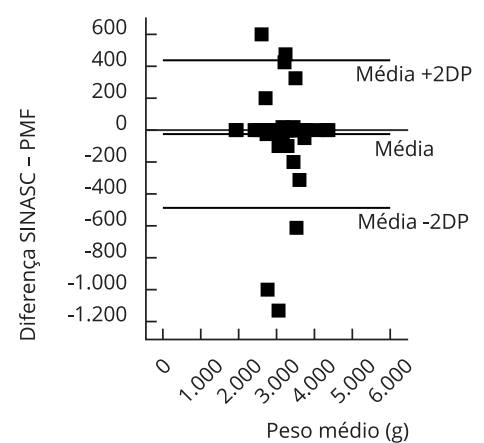

2b) Escolares com as quatro fontes de informação

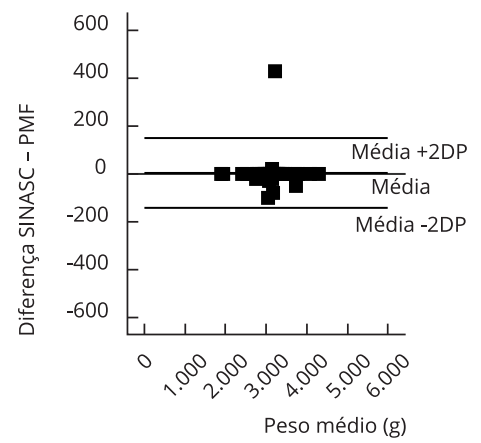

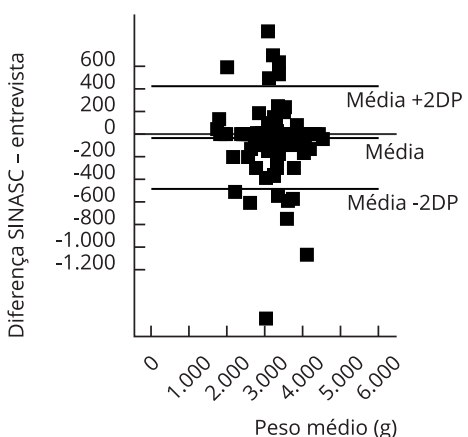
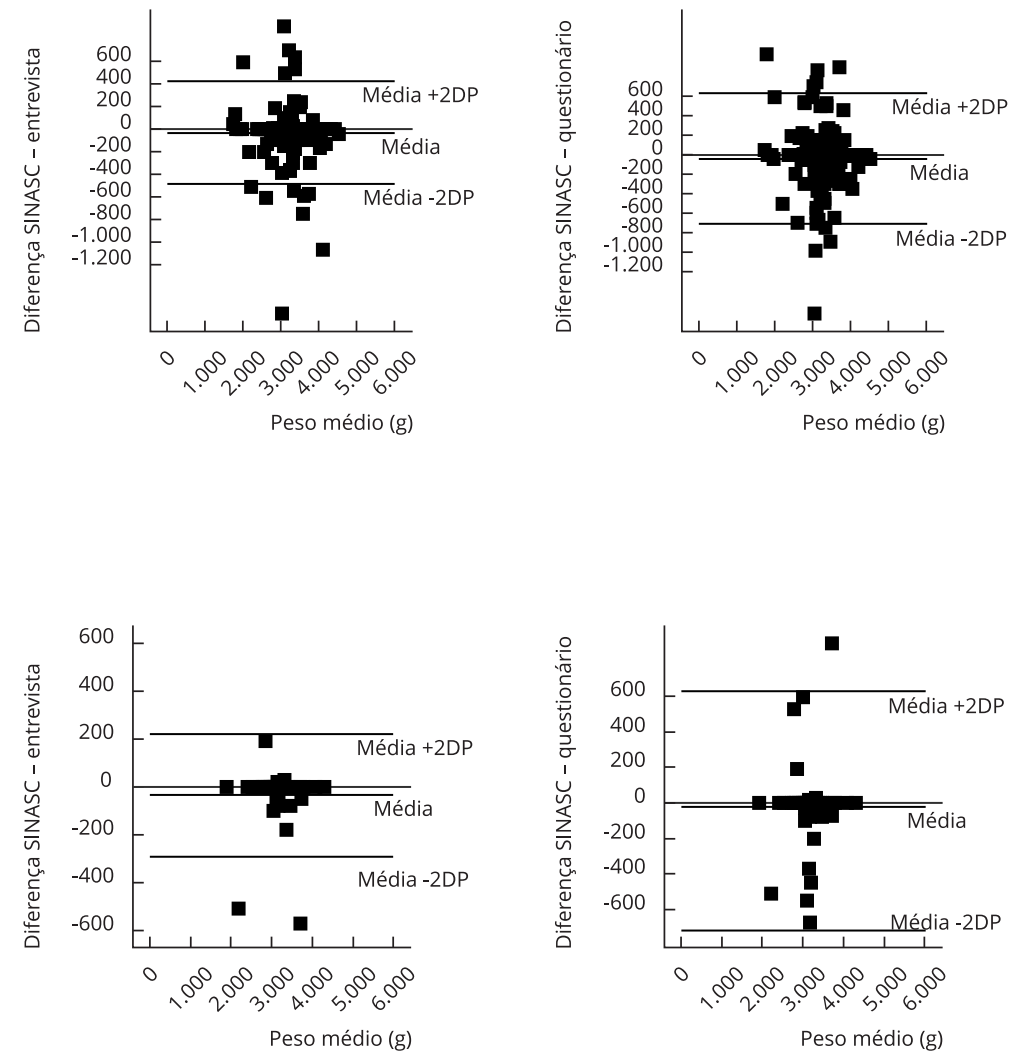

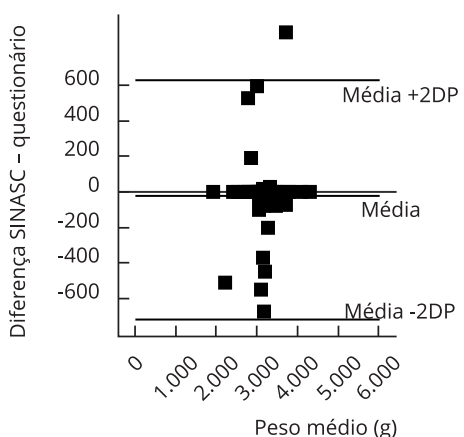

DP: desvio padrão; PMF: Programa Médico de Família; SINASC: Sistema de Informações sobre Nascidos Vivos. 


\section{Figura 3}

Gráficos de dispersão e coeficientes de correlação intraclasse (CCI) entre as fontes de informação de peso ao nascer dos escolares de 6-14 anos. Niterói, Rio de Janeiro, Brasil, 2010.

3a) Todos os escolares com, pelo menos, uma fonte de informação

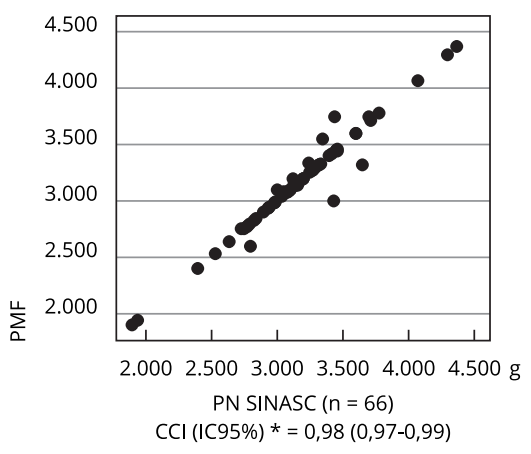

3b) Escolares com as quatro fontes de informação

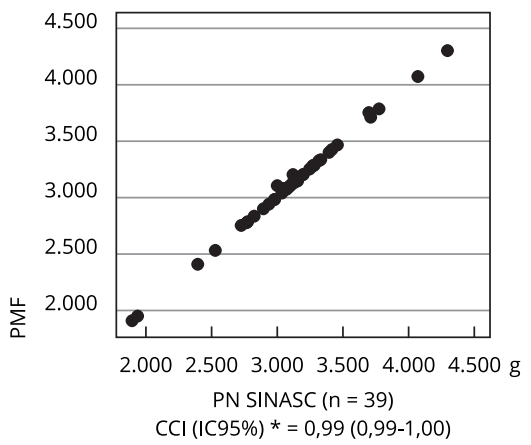

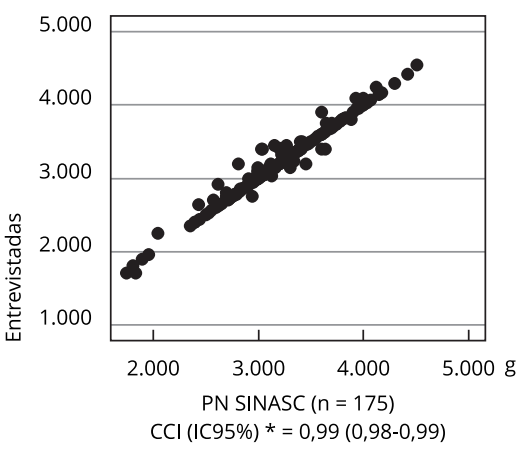
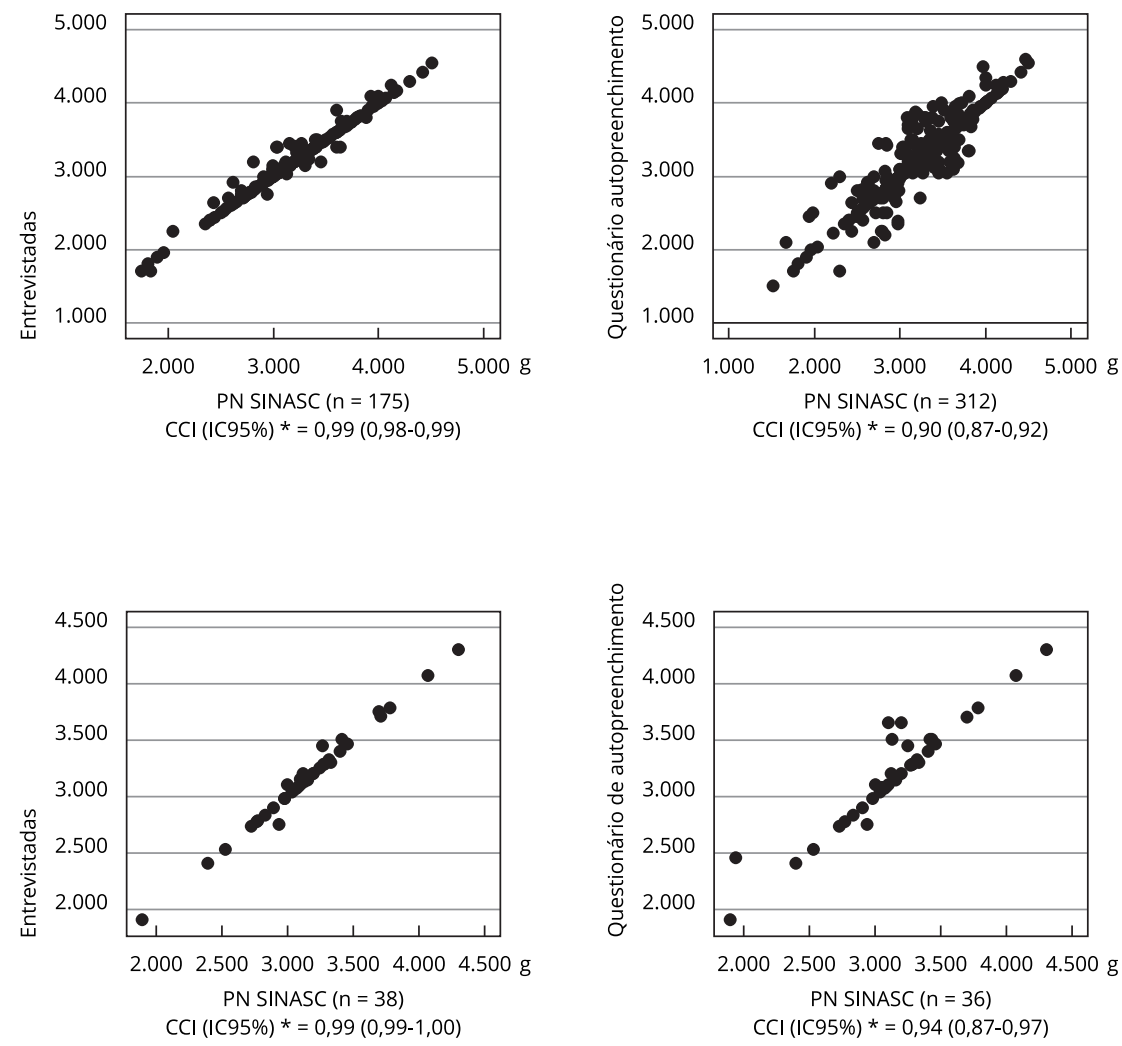

IC95\%: intervalo de 95\% de confiança; SINASC: Sistema de Informações sobre Nascidos Vivos; PMF: Programa Médico de Família.

* $\mathrm{CCl}$ individual (absolute agreement).

Os resultados da análise de tendenciosidade da informação do peso ao nascer entre as três fontes em relação ao SINASC são apresentados na Tabela 2. Foram excluídos 14 e 3 valores atípicos após a análise de resíduos da primeira etapa da modelagem, respectivamente, do grupo com todos os escolares e do grupo com os escolares com informações completas. No modelo final, independentemente da completude da informação do peso ao nascer nas quatro fontes (modelo A), a média de peso ao nascer no SINASC foi aproximadamente $3.196 \mathrm{~g}$, discretamente superior à média estimada no modelo B. Quando comparado ao SINASC, houve superestimação do peso ao nascer: as diferenças médias sistemáticas de peso ao nascer foram 3,5g no PMF, 18,4g na entrevista e cerca de $37 \mathrm{~g}$ no questionário de autopreenchimento, sendo esta última com significância estatística (valor de $p=0,001$ ). No modelo $\mathrm{B}$, correspondente ao grupo de estudantes com informações completas nas quatro fontes de informações, a média do peso ao nascer do SINASC foi cerca de 3.151g. O peso ao nascer registrado no PMF, na entrevista e no questionário de autopreenchimento apresentaram diferenças médias sistemáticas positivas de 8,4g, 9,7g e 8,1g, respectivamente, porém sem significância estatística. 
Tabela 2

Diferenças médias entre SINASC e as outras fontes de informação de peso ao nascer dos escolares de 6-14 anos total (Modelo A) e com as quatro fontes de informação (Modelo B). Niterói, Rio de Janeiro, Brasil, 2010.

\begin{tabular}{|c|c|c|c|}
\hline Fonte de informação do peso ao nascer & Diferença (g) & Valor de p & IC95\% \\
\hline \multicolumn{4}{|l|}{ Modelo A * } \\
\hline SINASC (referência) & $3.195,99$ & & \\
\hline PMF & 3,51 & 0,846 & $-31,99-39,02$ \\
\hline Entrevista & 18,42 & 0,152 & $-6,76-43,60$ \\
\hline Questionário ** & 36,95 & 0,000 & $15,67-58,24$ \\
\hline \multicolumn{4}{|l|}{ Modelo B *** } \\
\hline SINASC (referência) & $3.150,65$ & & \\
\hline PMF & 8,39 & 0,346 & $-9,05-25,82$ \\
\hline Entrevista & 9,68 & 0,277 & $-7,76-27,11$ \\
\hline Questionário & 8,07 & 0,365 & $-9,37-25,50$ \\
\hline
\end{tabular}

IC95\%: intervalo de 95\% de confiança; PMF: Programa Médico de Família; SINASC: Sistema de

Informações sobre Nascidos Vivos.

Questionário de autopreenchimento.

* 646 escolares e 1.329 observações;

** Questionário de autopreenchimento;

*** 31 escolares e 124 observações.

\section{Discussão}

Nesta investigação, foi observado que a informação do peso ao nascer coletada de diferentes fontes tem bom nível de confiabilidade. A validade de tal informação, apesar das diferenças médias sistemáticas do peso ao nascer apontarem uma discreta superestimação em relação ao SINASC, também foi considerada boa. Depois do SINASC, as fontes de informação do peso ao nascer mais fidedignas foram primeiramente o PMF, seguido da entrevista e do questionário de autopreenchimento.

Em relação aos registros do peso ao nascer de bases de dados nacionais e oficiais, estudos de confiabilidade da informação do peso ao nascer do SINASC foram realizados em maternidades do Sudeste e Nordeste, indicando uma excelente concordância 25,26,27,28. Apesar de a informação do peso ao nascer de prontuários de saúde da família ser utilizada para o cálculo de indicadores de avaliação do programa 29 , nenhum estudo de confiabilidade foi encontrado na literatura.

A ausência de informação do peso ao nascer exato neste estudo diferiu por faixa etária, com maior frequência entre adolescentes de 10-14 anos de idade, o que pode refletir tanto falhas nos sistemas de informações na sua fase imediata após a implantação quanto viés de memória. A informação recordatória de peso ao nascer foi realizada pela aplicação do questionário de autopreenchimento e por meio de entrevista. A maior variabilidade dos dados de peso ao nascer ocorreu com o questionário de autopreenchimento, em comparação com as outras fontes de dados. Observou-se também menor concordância e maior tendenciosidade à superestimação do peso ao nascer quando o par de dados incluía o questionário de autopreenchimento como fonte de informação, sugerindo, portanto, menor fidedignidade.

Alguns estudos nacionais 7,20 realizados na cidade de Pelotas, Rio Grande do Sul, baseados em coortes de nascimento, mostraram boa concordância e acurácia da informação recordatória sobre o peso ao nascer. A informação do peso ao nascer da coorte de nascimento de 1982 foi coletada na maternidade no momento do nascimento da criança e, depois, em entrevista domiciliar com a mãe da criança entre 9 e 15 meses de idade. A diferença absoluta do peso ao nascer foi menor do que $100 \mathrm{~g}$ em cerca de $80 \%$ das respostas 20 . Esse resultado é semelhante ao encontrado no presente estudo, considerando as fontes de informação sobre o peso ao nascer do PMF e SINASC. Na coorte de nascimento de 1993, em Pelotas, após 11 anos do nascimento, uma a cada quatro mães entrevistadas relatou diferenças absolutas do peso ao nascer exato maior que 200g. Nesse estudo, mães de crianças com peso 
ao nascer inferior a $3.500 \mathrm{~g}$ tenderam a superestimá-lo na entrevista, enquanto mães de crianças com peso igual ou superior a $3.500 \mathrm{~g}$ tenderam a subestimá-lo. A diferença média do peso ao nascer dessas duas fontes de informação foi de apenas $20 \mathrm{~g} 7$.

Estudos internacionais também apontaram uma boa concordância da informação recordatória. No estudo realizado no Tennessee (Estados Unidos) 30, entre os anos 1975 e 1984, foram avaliados 46.637 registros de nascimento e, em $70 \%$ dos casos, a concordância do peso ao nascer na certidão de nascimento foi exata ao relatado pela mãe em consulta subsequente ao nascimento (consultas ocorridas antes de um ano ou após um ano de idade da criança). Em Odense, Dinamarca 31, compararam-se o peso ao nascer do registro oficial de nascimentos e a informação relatada pelas mães de escolares, entre oito e dezoito anos, em questionário de autopreenchimento, mostrando que, em $92 \%$ dos casos, a diferença foi menor que $100 \mathrm{~g}$.

Robles \& Goldman ${ }^{9}$ descreveram alguns erros substanciais nos relatos de peso ao nascer obtidos por entrevistas a mães: relatos de peso ao nascer de crianças que provavelmente não foram pesadas no nascimento (principalmente as que pariram em casa); relatos de peso de uma consulta subsequente ao parto como se fosse peso ao nascer; e agrupamento de peso ao nascer, com uso de números inteiros. No entanto, vale ressaltar que essas conclusões foram oriundas de uma avaliação de pesquisas realizadas em países em desenvolvimento na América Latina (Guatemala, Bolívia, Costa Rica, El Salvador e Peru), com altas proporções de mulheres que viviam em áreas rurais e de crianças que não foram pesadas ao nascer. Além disso, há diferenças importantes na metodologia empregada, como a ausência de fontes de dados confiáveis para a comparação dos valores de peso ao nascer. Os resultados do presente estudo mostraram que as diferenças sistemáticas da média do peso ao nascer das três fontes de informação com o SINASC tenderam a superestimá-lo. A superestimação do peso ao nascer pode acarretar uma subestimação da frequência de baixo peso ao nascer $(<2.500 \mathrm{~g})$ e, consequentemente, enfraquecer associações negativas entre peso ao nascer e, por exemplo, obesidade 32,33. Considerando-se a pequena magnitude e a ausência de significância estatística para a maioria de tais diferenças no presente estudo, acredita-se que a superestimação encontrada não seja suficiente para inviabilizar, com esta base de dados, estudos de associação fundamentados na programação fetal.

Alguns resultados do presente estudo mostraram maior concordância entre o SINASC e a entrevista, enquanto outros mostraram uma menor tendenciosidade e maior concordância entre os dados do SINASC e os do PMF. Como os dados do PMF são dados coletados por profissionais da saúde e mais próximos ao momento do nascimento, foi dada prioridade na escolha dos dados do PMF em relação aos oriundos da entrevista.

Como fatores limitantes do presente estudo, podem ser relatados: a não aleatoriedade da amostra; a não inscrição de todas as crianças no PMF, pois aproximadamente $56 \%$ dos escolares não relataram a unidade de saúde em que estavam inscritos, o que dificultou a busca pelo prontuário; o questionário de autopreenchimento e a entrevista poderiam ser respondidos por um responsável do escolar, preferencialmente, mas não obrigatoriamente, pela mãe biológica, que a princípio estaria mais apta a responder do que pais adotivos ou parentes próximos; e a não obrigatoriedade do nome completo da mãe no SINASC até o ano de 1997, o que limitou o relacionamento das bases de dados dos escolares mais velhos. É importante destacar pontos positivos do nosso estudo. Foram apresentados dados sobre o peso ao nascer oriundos de fontes oficiais com informações do nascido vivo, como SINASC e prontuários do PMF, preenchidos próximo ao nascimento. Esses dados oficiais foram comparados com informações colhidas de 6-14 anos após o nascimento do escolar. A confiabilidade aceitável da informação sobre o peso ao nascer após esse longo intervalo de tempo é uma importante vantagem para os estudos de programação fetal ou estudos focalizados no ciclo da vida que necessitam de informações sobre o nascimento.

As fontes oficiais de informações perinatais, como SINASC e prontuários do PMF, nas quais o peso ao nascer é registrado próximo ao nascimento, representam uma vantagem para os estudos de programação fetal. Na ausência das mesmas, a lembrança do peso ao nascer de seis a catorze anos após o nascimento representou uma opção confiável no nosso estudo e permitiu completar a informação sobre peso ao nascer exato de $33 \%$ da população de estudo. A informação retrospectiva obtida por questionário de autopreenchimento mostrou-se mais frágil do que a obtida por entrevista, apresentando uma diferença, estatisticamente significante, da média de peso ao nascer entre o questionário de autopreenchimento e o SINASC. No entanto, recomenda-se que, na ausência de qualquer outra 
fonte de informação, esta seja utilizada, já que a diferença da média de peso ao nascer encontrada foi menor do que $100 \mathrm{~g}$ (cerca de $37 \mathrm{~g}$ no grupo de todos os escolares e $8 \mathrm{~g}$ no grupo com as informações completas), que já foi reconhecida na literatura como uma diferença aceitável 20.

Assim, para completar a informação do peso ao nascer dos escolares participantes da pesquisa que não tinham a informação do SINASC, foi estabelecida uma ordenação decrescente de confiabilidade: (1) PMF; (2) entrevista; e (3) questionário de autopreenchimento.

\section{Colaboradores}

G. A. Noronha, P. L. Kale, T. Z. G. Torres e M. Szklo participaram de todas as etapas da elaboração do artigo. A. J. L. Costa e M. L. T. Cavalcanti participaram da discussão dos resultados e da redação do artigo.

\section{Agradecimentos}

Os autores agradecem ao Conselho Nacional de Desenvolvimento Científico e Tecnológico (CNPq; Edital MCT/CNPq 15/2007, Universal; no 481094/2007-5), à Fundação Carlos Chagas Filho de Amparo à Pesquisa no Estado do Rio de Janeiro (Faperj; Edital de Apoio às Instituições de Ensino e Pesquisa Sediadas no Estado do Rio de Janeiro: E-26 110.986/2008) e à Financiadora de Estudos e Projetos (FINEP) Encomenda Vertical de Projeto de Pesquisa (referência 0436/08) pelo apoio financeiro.

\section{Referências}

1. Barker DJ, Osmond C. Infant mortality, childhood nutrition, and ischaemic heart disease in England and Wales. Lancet 1986; 10:1077-81.

2. Victora CG, González DA, Hallal PC. Epidemiologia do ciclo vital. In: Almeida Filho N, Barreto ML, organizadores. Epidemiologia \& saúde: fundamentos, métodos, aplicações. Rio de Janeiro: Editora Guanabara Koogan; 2012. p. 438-46.

3. Perry IJ, Lumey LH. Fetal growth and development: the role of nutrition and other factors. In: Kuh D, Ben-Shlomo Y, editors. Life course approach to chronic disease epidemiology. 2nd Ed. Oxford: Oxford University Press; 2004. p. 345-70.

4. Boguszewski MCS, Mericq V, Bergada I, Damiani D, Belgorosky A, Gunczler P, et al. Latin American consensus: children born small for gestational age. BMC Pediatr 2011; 11:66.

5. Kiely JL, Brett KM, Yu S, Rowley DL. Low birthweight and intrauterine growth retardation. In: Wilcox LS, Marks JS, editors. From data to action: CDC's public health surveillance for women, infants, and children. Atlanta: Centers for Disease Control and Prevention; 1994. p. 185-202.

6. Adams MM. Birth weight, gestational duration, and fetal growth. In: Adams MM, Alexander GR, Kirby RS, Wingate MS, editors. Perinatal epidemiology for public health practice. New York: Springer; 2009. p. 225-73.

7. Araújo CLP, Dutra CLC, Hallal PC. Validity of maternal report on birth weight 11 years after delivery: the 1993 Pelotas Birth Cohort Study, Rio Grande do Sul State, Brazil. Cad Saúde Pública 2007; 23:2421-7.

8. Lucia VC, Luo Z, Gardiner JC, Paneth N, Breslau N. Reports of birthweight by adolescents and their mothers: comparing accuracy and identifying correlates. Paediatr Perinat Epidemiol 2006; 20:520-7.

9. Robles A, Goldman N. Can accurate data on birthweight be obtained from health interview surveys? Int J Epidemiol 1999; 28:925-31.

10. Mbuagbaw L, Gofin R. Can recall of birth size be used as a measure of birthweight in Cameroon? Paediatr Perinat Epidemiol 2010; 24:383-9. 
11. Szklo M, Nieto FJ. Understanding lack of validity: bias. In: Szklo M, Javier Nieto F, editors. Epidemiology: beyond the basics. 3rd Ed. Burlington: Jones \& Bartlett Learning; 2014. p. 109-52.

12. Lobato JCP, Costa AJL, Kale PL, Cavalcanti MLT, Kuschnir MCC, Velarde LGC, et al. Programação fetal e alterações metabólicas em escolares: metodologia de um estudo caso-controle. Rev Bras Epidemiol 2016; 19:52-62.

13. Almeida MF, Alencar GP, França Jr. I, Novaes HMD, Siqueira AAF, Schoeps D, et al. Validade das informações das declarações de nascidos vivos com base em estudo de caso-controle. Cad Saúde Pública 2006; 22:643-52.

14. Guimarães PV, Coeli CM, Cardosoi RCA, Medronho RA, Fonseca SC, Pinheiro RS. Confiabilidade dos dados de uma população de muito baixo peso ao nascer no Sistema de Informações sobre Nascidos Vivos 2005-2006. Rev Bras Epidemiol 2012; 15:694-704.

15. Mello-Jorge MHP, Gotileb SLD, Soboll MLMS, Almeida MF, Latorre MRDO. Avaliação do Sistema de Informações sobre Nascidos Vivos e o uso de seus dados em epidemiologia e estatísticas de saúde. Rev Saúde Pública 2001; 35:508-14.

16. Mello Jorge MHP, Laurenti R, Gotlieb LSD. Análise da qualidade das estatísticas vitais brasileiras: a experiência de implantação do SIM e do SINASC. Ciênc Saúde Coletiva 2007; 12:643-54.

17. Ministério da Saúde. Sistema de Informação sobre Nascidos Vivos - SINASC. Inf Epidemiol SUS 1992; I:5-16.

18. Kale PL. Primeira avaliação do Sistema de Informações sobre Nascidos Vivos no município de Niterói - 1992. Cad Saúde Colet (Rio J.) 1997; 5:53-64.

19. Hübner LCM, Franco TB. O Programa Médico de Família de Niterói como estratégia de implementação de um modelo de atenção que contemple os princípios e diretrizes do SUS. Physis (Rio J.) 2007; 17:173-91.

20. Victora CG, Barros FC, Martines JC, Béria JU, Vaughan JP. As mães lembram o peso ao nascer de seus filhos? Rev Saúde Pública 1985; 19:195-200.

21. Bland JM, Altman DG. Statistical methods for assessing agreement between two methods of clinical measurements. Lancet 1986; 327: 307-10.

22. Shrout PE, Fleiss JL. Intraclass correlations: uses in assessing rater reliability. Psychol Bull 1979; 86:420-8.
23. McGraw KO, Wong SP. Forming inferences about some intraclass correlation coefficients. Psychol Methods 1996; 1:30-46.

24. Maranhão Neto GA, Leon ACMP, Farinatti PTV. Equivalência transcultural de três escalas utilizadas para estimar a aptidão cardiorrespiratória: estudo em idosos. Cad Saúde Pública 2008: 24:2499-510.

25. Gabriel GP, Chiquetto L, Morcillo AM, Ferreira MC, Bazan IGM, Daolio LD, et al. Avaliação das informações das Declarações de Nascidos Vivos do Sistema de Informação sobre Nascidos Vivos (SINASC) em Campinas, São Paulo, 2009. Rev Paul Pediatr 2014; 32:183-8.

26. Mascarenhas MDD, Gomes KRO. Confiabilidade dos dados do Sistema de Informações sobre Nascidos vivos em Teresina, Estado do Piauí, Brasil - 2002. Ciênc Saúde Coletiva 2011; 16 Suppl 1:1233-9.

27. Silva AAM, Ribeiro VS, Borba Júnior AF, Coimbra LC, Silva RA. Avaliação da qualidade dos dados do Sistema de Informações sobre Nascidos Vivos em 1997-1998. Rev Saúde Pública 2001; 35:508-14.

28. Theme Filha MM, Gama SGN, Cunha CB, Leal MC. Confiabilidade do Sistema de Informações sobre Nascidos Vivos Hospitalares no Município do Rio de Janeiro, 1999-2001. Cad Saúde Pública 2004; 20 Suppl 1:S83-91.

29. Mendoza-Sassi RA, Cesar JA, Teixeira TP, Ravache C, Araújo GD, Silva TC. Diferenças no processo de atenção ao pré-natal entre unidades da Estratégia Saúde da Família e unidades tradicionais em um município da Região Sul do Brasil. Cad Saúde Pública 2011; 27:787-96.

30. Gayle HD, Yip R, Frank MJ, Nieburg P, Binkin NJ. Validation of maternally reported birth weights among 46,637 Tennessee WIC Program participants. Public Health Rep 1988; 103:143-7.

31. Adegboye ARA, Heitmannb BL. Accuracy and correlates of maternal recall of birthweight and gestational age. BJOG 2008; 115:886-93.

32. Boeke CE, Marín C, Oliveros H, Mora-Plazas M, Agudelo-Cañas S, Villamor E. Validity of maternal birthweight recall among Colombian children. Matern Child Health J 2012; 16:753-9.

33. Lia CY, Weib JN, Lud TH, Chuangc LM, Sung FC. Mothers tended to overreport categorical infant birth weight of their children. J Clin Epidemiol 2006; 59:1319-25. 


\section{Abstract}

Birth weight is essential information in fetal programming studies and is generally obtained retrospectively. In Brazil's Information System on Live Births (SINASC), birth weight is valid information but is not always accessible. The study aimed to establish an algorithm for the selection of the most reliable data source for birth weight in the absence of information in the SINASC database. In a cross-sectional study of 6-14-year-old schoolchildren in Niterói, Rio de Janeiro State, Brazil, in 2010, birth weight was collected through a self-completed questionnaire, interview, medical records from the Family Physician Program, and the SINASC database. We calculated intra-class correlation coefficients (ICCs) and differences in mean birth weight. ICCs varied from 0.90 to 0.99 . All the other sources showed higher mean birth weight than SINASC, allowing differences up to 100g. Birth weight is recorded at birth (SINASC) or close to it (Family Physician Program), and in the absence of these sources, birth weight as retrieved at 6-14 years of age is a reliable option. To complement information on birth weight in the absence of SINASC, we recommend the following order: Family Physician Program, interview, and questionnaire.

Birth Weight; Reproducibility of Results; Information Systems; Fetal Development

\section{Resumen}

El peso al nacer es una información esencial en los estudios de programación fetal y, en general, obtenida retrospectivamente. En el Sistema de Información sobre Nacidos Vivos (SINASC), peso al nacer es una información válida, pero no siempre accesible. Tuvo como objetivo establecer un algoritmo para la selección de la fuente de datos de mayor confiabilidad del peso al nacer, en ausencia de información del SINASC. En el estudio por secciones de estudiantes de 6-14 años, Niterói, Río de Janeiro, Brasil, 2010, el peso al nacer se recogió mediante un cuestionario para autocompletar, entrevista, historias clínicas del Programa Médico de Familia (PMF), además del SINASC. Se calcularon coeficientes de correlación intraclase (CCI) $y$ diferencias de las medias de peso al nacer. Los CCIs variaron de 0,90 a 0,99. Todas las fuentes presentaron medias de peso al nacer mayores que el SINASC, considerándose aceptables diferencias hasta 100g. El peso al nacer es registrado en el nacimiento (SINASC) o cercano a este (PMF) y, en la ausencia de estas fuentes, el recuerdo del peso al nacer de 6-14 años tras el nacimiento es una opción fiable. Para complementar la información sobre peso al nacer, en ausencia del SINASC, se recomienda la siguiente ordenación: PMF, entrevista y cuestionario.

Peso al Nacer; Reproducibilidad de Resultados; Sistemas de Información; Desarrollo Fetal
Recebido em 29/Mar/2016

Versão final reapresentada em 09/Ago/2016 Aprovado em 08/Set/2016 